\title{
Two Dogmas About Quantum Mechanics
}

\author{
Jeffrey Bub \\ Department of Philosophy, University of Maryland, College Park, MD 20742* \\ Itamar Pitowsky \\ Department of Philosophy, The Hebrew University, Jerusalem, Israel ${ }^{\dagger}$
}

\begin{abstract}
We argue that the intractable part of the measurement problem- the 'big' measurement problem - is a pseudo-problem that depends for its legitimacy on the acceptance of two dogmas. The first dogma is John Bell's assertion that measurement should never be introduced as a primitive process in a fundamental mechanical theory like classical or quantum mechanics, but should always be open to a complete analysis, in principle, of how the individual outcomes come about dynamically. The second dogma is the view that the quantum state has an ontological significance analogous to the significance of the classical state as the 'truthmaker' for propositions about the occurrence and non-occurrence of events, i.e., that the quantum state is a representation of physical reality. We show how both dogmas can be rejected in a realist information-theoretic interpretation of quantum mechanics as an alternative to the Everett interpretation. The Everettian, too, regards the 'big' measurement problem as a pseudo-problem, because the Everettian rejects the assumption that measurements have definite outcomes, in the sense that one particular outcome, as opposed to other possible outcomes, actually occurs in a quantum measurement process. By contrast with the Everettians, we accept that measurements have definite outcomes. By contrast with the Bohmians and the GRW 'collapse' theorists who add structure to the theory and propose dynamical solutions to the 'big' measurement problem, we take the problem to arise from the failure to see the significance of Hilbert space as a new kinematic framework for the physics of an indeterministic universe, in the sense that Hilbert space imposes kinematic (i.e., pre-dynamic) objective probabilistic constraints on correlations between events.
\end{abstract}

\section{Oxford Everett}

The salient difference between classical and quantum mechanics is the noncommutativity of the operators representing the physical magnitudes ('observables') of a quantum mechanical system - or, equivalently, the transition from a classical event space, represented by the Boolean algebra of (Borel) subsets of a phase space, to a non-Boolean

\footnotetext{
*E-mail address: jbub@umd.edu

${ }^{\dagger}$ E-mail address: itamarp@vms.huji.ac.il
} 
quantum event space represented by the projective geometry of closed subspaces of a Hilbert space, which form an infinite collection of intertwined Boolean algebras, each Boolean algebra corresponding to a resolution of the identity: a partition of the Hilbert space representing a family of mutually exclusive and collectively exhaustive events.

Probabilities in quantum mechanics are, as von Neumann put it [32, p. 245], 'uniquely given from the start' as a nonclassical relation between events represented by the angles in the projective geometry of subspaces of Hilbert space. If $e$ and $f$ are atomic (elementary) events, the 'transition probability' (Born probability) between the events is:

$$
\operatorname{prob}(e, f)=|\langle e \mid f\rangle|^{2}=|\langle f \mid e\rangle|^{2}=\cos ^{2} \theta_{e f}
$$

The transition probability can be expressed as:

$$
\operatorname{prob}_{e}(f)=\operatorname{Tr}\left(P_{e} P_{f}\right)
$$

where $P_{e}$ and $P_{f}$ are the projection operators onto the 1-dimensional subspaces representing the events $e$ and $f$, respectively. Uniqueness is shown by Gleason's theorem [17]: ${ }^{1}$ in a Hilbert space $\mathcal{H}$ of dimension greater than 2 , if $\sum_{i} \operatorname{prob}\left(f_{i}\right)=1$ for the atomic events $f_{i}$ in each Boolean algebra generated by a partition of the Hilbert space into orthogonal 1-dimensional subspaces, then the probabilities of events $f$ represented by subspaces of $\mathcal{H}$ are uniquely represented as:

$$
\operatorname{prob}_{\rho}(f)=\operatorname{Tr}\left(\rho P_{f}\right)
$$

where $P_{f}$ is the projection operator onto the subspace representing the event $f$ and $\rho$ is a density operator representing a pure state $\left(\rho=P_{e}\right.$, for some atomic event $e$ ) or a mixed state $\left(\rho=\sum_{i} w_{i} P_{e_{i}}\right)$. It is assumed that the assignment of probabilities satisfies a condition that Barnum et al [4] call 'the noncontextuality of probability,' that the probability assigned to an event $f$ depends only on $f$ and is independent of the Boolean algebra to which the event belongs. Note that if ' $f$ in context 1 ' and ' $f$ in context 2' represented two distinct events, we could not represent the structure of quantum events as the projective geometry of subspaces of a Hilbert space: we would have to enlarge the structure.

The question is: what do these 'transition probabilities' or 'transition weights' mean? The probabilities are probabilities of — what? Evidently, $|\langle e \mid f\rangle|^{2}$ does not represent the probability of a spontaneous transition from an event $e$ to the event $f$. The textbook answer is that $|\langle e \mid f\rangle|^{2}$ represents the probability, for a system in the state $|e\rangle$ in which the event $e$ has probability 1, of finding the event $f$ in a measurement of an observable of the system, where the set of possible outcomes of the measurement generates a Boolean algebra, representing a partition of the Hilbert space containing the event $f$ (but note, not the event $e$ ).

The textbook answer by itself, without adding anything more to the story of how these events are supposed to come about in a measurement process, is adequate only if we are content with an instrumentalist interpretation of the theory. Why? The structure

\footnotetext{
${ }^{1}$ For von Neumann, uniqueness is a consequence of invariance under the unitary symmetries of the projective lattice representing events.
} 
of the quantum event space determines the kinematic part of quantum theory. This includes the association of Hermitian operators with observables, the Born probabilities, the von Neumann-Lüders conditionalization rule, and the unitarity constraint on the dynamics, which is related to the event structure via a theorem of Wigner [37],[31]. The transition from the state $|e\rangle$, in which the event $e$ has probability 1, to the state $|f\rangle$, in which the event $f$ has probability 1 , with probability $|\langle e \mid f\rangle|^{2}$ in a measurement process is a non-unitary stochastic transition that is not described by the unitary dynamics. Since the probability of the event $e$ was 1 before the measurement and is now, in the state $|f\rangle$ after the occurrence of the measurement outcome $f$, less than 1, there is a loss of information on measurement or-as Bohr put it—an 'irreducible and uncontrollable' measurement disturbance. Without a dynamical explanation of this measurement disturbance, or an analysis of what is involved in a quantum measurement process that addresses the issue (including, possibly, rejecting the 'eigenvalue-eigenstate rule'-the association of the outcome event $f$ with the state $|f\rangle$-as in Bohm's theory or modal interpretations), the theory qualifies as an algorithm for predicting the probabilities of measurement outcomes, but cannot be regarded as providing a realist account, in principle, of how events unfold in a measurement process.

This is the measurement problem. Proposed solutions to the problem, such as Bohm's 'hidden variable' theory [18] or the GRW 'dynamical collapse' theory [16], add structure to the theory: particle trajectories in the case of Bohm's theory or a nonunitary stochastic dynamics for the quantum state in the case of the GRW theory. The Everett interpretation purports to solve the problem without adding any new structural elements to quantum mechanics.

The central claims of the Everett interpretation in the 'Oxford' version developed by Deutsch [13], Saunders [28, 29, 30], Wallace [34, 33, 35, 36], Greaves and others $[19,21,20]$ can be outlined as follows:

Ontology At the most fundamental level, what there is is described by the quantum state of the universe- so whatever is true or false is determined by the quantum state as the 'truthmaker' for propositions about the occurrence and nonoccurrence of events.

Branching A family of effectively non-interfering or decoherent histories of coarsegrained events associated with relatively stable systems at the macrolevel emerges through the dynamical process of decoherence, as a consequence of the Hamiltonians that characterize the dynamical evolution of the universal quantum state. With respect to the coarse-grained basis selected by decoherence, the quantum state decomposes into a linear superposition that can be interpreted as describing an emergent branching structure of non-interfering quasi-classical histories or 'worlds,' identified with the familiar classical macroworlds of our experience, weighted by the Born probabilities. The alternative outcomes of a quantum measurement process are associated with different branches in the decomposition of the quantum state with respect to the decoherence basis. There is no fact of the matter as to the number of branches: the history space is a quasi-classical probability space that is inherently vaguely defined (appropriately so, given the vague specification of macro-configurations). The coarse-graining of the event 
space can be refined or coarsened to a certain extent without compromising effective decoherence, and the decoherence basis can be unitarily transformed (e.g., rotated) over a certain range of transformations without compromising decoherence.

Uncertainty/Caring There is a sense in which a rational agent on a branch, faced with subsequent branching, can be uncertain about the future (i.e., uncertain about 'which branch the agent will subsequently occupy'). Such an agent can have rational credences (degrees of belief that satisfy the axioms of probability theory) about the outcomes of quantum measurements, even though all outcomes occur on different branches. Alternatively, even without uncertainty, an agent faced with multiple futures will care about what happens on a branch, and so will have a 'caring measure' for decision-making that quantifies the extent of caring for different branches and satisfies the axioms of probability theory.

Probability To achieve a realist interpretation of quantum mechanics that solves the measurement problem, it suffices to postulate that an agent's credence function or caring measure conforms to the objective quantum mechanical weights of the different branches. In fact, it is possible to prove that this must be so, given standard rationality constraints on an agent's preferences, and a measurement neutrality assumption: that a rational agent is indifferent between two quantum wagers that agree on the quantum state, the observable measured, and the payoff function on the outcomes, i.e., the agent is indifferent between alternative measurement procedures; alternatively, the result follows from a related equivalence assumption: that a rational agent assigns equal credences to events that are assigned equal quantum weights. These additional assumptions can be justified as rationality constraints, but only on the Everett interpretation, in which all possible measurement outcomes occur, relative to different branches.

The Everettian aims to show that standard quantum mechanics can be understood as a complete theory in a realist sense-that the measurement problem does not reduce the theory to an instrument for the probabilistic prediction of measurement outcomes. The basic problem for the Everettian is to 'save the appearances,' given the radical difference between our experience of a stable macroworld and the ontological assumption. The dynamics of decoherence yields an emergent weighted branching structure of quasi-classical histories at the macrolevel. So what has to be explained is how uncertainty or caring makes sense when all alternatives occur relative to different branches, and how the quantum weights - which are a feature of the quantum state, i.e., the ontology - are associated with the credence function or caring measure of rational agents. The measurement problem is the problem of explaining the apparently 'irreducible and uncontrollable disturbance' in a quantum measurement process, the 'collapse' of the wave function described by von Neumann's projection postulate. The Everettian's solution is to show how appearances can be saved by denying that there is any such disturbance, on the basis that no definite outcome is selected in a measurement-all outcomes are selected relative to different branches, according to the quantum theory. The appearance of disturbance on a single branch is a reflection of how the quantum weights are distributed in the emergent process of branching, and 
if we either assume or prove that our credence function or caring measure should conform to these weights, then we have an explanation for the appearance of disturbance in a realist interpretation of quantum mechanics as a complete dynamical theory.

Of course, everything hinges on whether the different components of the intepretation can be established satisfactorily, and there is now an extensive literature challenging and defending these claims, especially Uncertainty/Caring and Probability. Here we simply list these components ${ }^{2}$ and note that the claim is that the Everett interpretation solves the measurement problem on the basis of (i) the weighted branching structure of quasi-classical histories that emerges through the dynamical process of decoherence, (ii) an argument that rational agents can be uncertain or care differently about different futures in a branching universe, and (iii) the proposal that the credence function or caring measure of rational agents should conform to the weights of the branches. For the Everettian, the icing on the cake is that the interpretation yields a derivation of Lewis's Principal Principle: the identification of an objective feature of the world - the quantum weights - with the credence function or caring measure of rational agents, and hence the interpretation of the quantum weights as objective chances. But the cake itself, so to speak, is independent of this additional feature. (See Wallace [36].)

In a previous publication [27], one of us characterized debates about the foundations of quantum mechanics in terms of two assumptions or dogmas, and distinguished two measurement problems: a 'big' measurement problem and a 'small' measurement problem. The first dogma is Bell's assertion (defended in [6]) that measurement should never be introduced as a primitive process in a fundamental mechanical theory like classical or quantum mechanics, but should always be open to a complete analysis, in principle, of how the individual outcomes come about dynamically. The second dogma is the view that the quantum state has an ontological significance analogous to the ontological significance of the classical state as the 'truthmaker' for propositions about the occurrence and non-occurrence of events, i.e., that the quantum state is a representation of physical reality. The 'big' measurement problem is the problem of explaining how measurements can have definite outcomes, given the unitary dynamics of the theory: it is the problem of explaining how individual measurement outcomes come about dynamically. The 'small' measurement problem is the problem of accounting for our familiar experience of a classical or Boolean macroworld, given the non-Boolean character of the underlying quantum event space: it is the problem of explaining the dynamical emergence of an effectively classical probability space of macroscopic measurement outcomes in a quantum measurement process.

The 'big' measurement problem depends for its legitimacy on the acceptance of the two dogmas. We argue below that both dogmas should be rejected, and that the 'big' measurement problem is a pseudo-problem. In a sense, the Everettian, too, regards the 'big' measurement problem as a pseudo-problem, because the Everettian rejects the assumption that measurements have definite outcomes, in the sense that one particular outcome, as opposed to other possible outcomes, actually occurs in a quantum measurement process. By contrast with the Everettians, we accept that measurements have definite outcomes. By contrast with the Bohmians and the GRW 'collapse' theorists

${ }^{2}$ For a critique of Probability by one of us, see Hemmo and Pitowsky [22]. 
who add structure to the theory and propose dynamical solutions to the 'big' measurement problem, we take the problem to arise from the failure to see the significance of Hilbert space as a new kinematic framework for the physics of an indeterministic universe, in the sense that Hilbert space imposes kinematic (i.e., pre-dynamic) objective probabilistic constraints on correlations between events. By 'predynamic' here, we refer to generic features of quantum systems, independent of the details of the dynamics (see Jannsen [24] for a similar kinematic-dynamic distinction in the context of special relativity). The 'small' measurement problem is resolved by considering the dynamics of the measurement process and the role of decoherence in the emergence of an effectively classical probability space of macro-events to which the Born probabilities refer (alternatively, by considering certain combinatorial features of the probabilistic structure: see Pitowsky [27, §4.3]).

In the following section, we list the essential features of the proposed informationtheoretic interpretation, somewhat more extensively than our brief sketch of the Everett interpretation. Further discussion follows in a subsequent Commentary.

\section{An Information-Theoretic Interpretation of Quantum Mechanics}

The elements of the information-theoretic interpretation we propose ${ }^{3}$ can be set out as follows:

'No Cloning' The empirical discovery underlying the transition from classical to quantum mechanics is the discovery that chance set-ups behave differently than we thought they did. More precisely: there are information sources that cannot be broadcast - there is no universal cloning machine capable of copying the outputs of an arbitrary information source.

Kinematics Hilbert space as a projective geometry (i.e., the subspace structure of Hilbert space) represents a non-Boolean event space, in which there are builtin, structural probabilistic constraints on correlations between events (associated with the angles between events) - just as in special relativity the geometry of Minkowski space-time represents spatio-temporal constraints on events. Certain principles characterizing physical processes motivate the choice of Hilbert space as the representation space for the correlational structure of events, just as Einstein's principle of special relativity and the light postulate motivate the choice of Minkowski space-time as the representation space for the spatio-temporal structure of events. In the case of quantum mechanics, these principles are information-theoretic and include a 'no signaling' principle and a 'no cloning' principle. The structure of Hilbert space imposes kinematic (i.e., pre-dynamic) objective probabilistic constraints on events to which a quantum dynamics of matter and fields is required to conform, through its symmetries, just as the structure of Minkowski space-time imposes kinematic constraints on events to which a relativistic dynamics is required to conform. In this sense Hilbert space

${ }^{3}$ For related views, see Demopoulos [12], Pitowsky [26, 27]. 
provides the kinematic framework for the physics of an indeterministic universe, just as Minkowski space-time provides the kinematic framework for the physics of a non-Newtonian, relativistic universe. There is no deeper explanation for the quantum phenomena of interference and entanglement than that provided by the structure of Hilbert space, just as there is no deeper explanation for the relativistic phenomena of Lorentz contraction and time dilation than that provided by the structure of Minkowski space-time.

Dynamics The unitary quantum dynamics evolves the whole structure of events with probabilistic correlations in Hilbert space (in the Heisenberg picture), not the evolution from one configuration of the universe to another, i.e., not the evolution from one actual co-occurrence of events to a subsequent actual co-occurrence of events. This means that there can be a real change in the correlations between events at the microlevel without a change in the occurrence of events at the macrolevel (as in the evolution of a quantum system through the unitary gates of a quantum computer, prior to the final measurement).

Probability By Gleason's theorem, there is a unique assignment of credences conforming to the structural probabilistic constraints (the objective chances) of Hilbert space (see Pitowsky [26]). These credences are encoded in the quantum state. So the quantum state is a credence function.

Information Loss The salient principle marking the transition from classical to nonclassical theories of information is the 'no cloning' principle: there is no universal cloning machine capable of copying the outputs of an arbitrary information source. ${ }^{4}$ This principle entails a loss of information in a measurement processan 'irreducible and uncontrollable disturbance'-irrespective of how the measurement process is implemented dynamically. The loss of information is to be understood, ultimately, as a kinematic effect of the nonclassical quantum event space, just as Lorentz contraction is, ultimately, a kinematic effect in special relativity.

Completeness Conditionalizing on a measurement outcome leads to a nonclassical updating of the credence function represented by the quantum state via the von Neumann-Lüders rule, which expresses the information loss on measurement. This updating is consistent with a dynamical account of the correlations between micro and macro-events in a quantum measurement process. The Hamiltonians characterizing the interactions between microsystems and macrosystems, and the interactions between macrosystems and their environment, are such that certain relatively stable structures of events associated with the familiar macrosystems of our experience emerge at the macrolevel, forming an effectively classical probability space. This amounts to a consistency proof that, say, a SternGerlach spin-measuring device or a bubble chamber behaves dynamically according to the kinematic constraints represented by the projective geometry of Hilbert space, as these constraints manifest themselves at the macrolevel. Such a consistency proof demonstrates the completeness of quantum mechanics. Given

${ }^{4}$ More precisely, there is no universal broadcasting machine. See below. 
the 'no cloning' principle underlying the kinematics of Hilbert space, there is no further story to be told about how individual measurement outcomes come about dynamically (assuming we don't add structure to the theory, such as Bohmian trajectories or dynamical 'collapses'). Similarly, the dynamical explanation of relativistic phenomena like Lorentz contraction in terms of forces, insofar as the forces are required to be Lorentz invariant, amounts to a consistency proof. There is no further story to be told about Lorentz contraction, once it is shown how to provide a dynamical account consistent with the kinematic constraints of Minkowski geometry (assuming we don't add structure to the theory, such as the ether).

Realism The possibility of a dynamical analysis of measurement processes consistent with the Hilbert space kinematic constraints justifies the information-theoretic interpretation of quantum mechanics as realist and not merely a predictive instrument for updating probabilities on measurement outcomes.

\section{Commentary}

On the information-theoretic interpretation, the quantum state is a credence function, a bookkeeping device for keeping track of probabilities - the universe's objective chancesnot the quantum analogue of the dynamically evolving classical state understood as the 'truthmaker' for propositions about the occurrence and non-occurrence of events.

Conditionalization on the occurrence of an event $a$, in the sense of a minimal revision - consistent with the subspace structure of Hilbert space- of the probabilistic information encoded in a density operator $\rho$, is given by the von Neumann-Lüders rule: ${ }^{5}$

$$
\rho \rightarrow \rho_{a} \equiv \frac{P_{a} \rho P_{a}}{\operatorname{Tr}\left(P_{a} \rho P_{a}\right)}
$$

where $P_{a}$ is the projection operator onto the subspace representing the event $a$. That is, $\rho_{a}$ is the conditionalized density operator, conditional on the event $a$, and the normalizing factor $\operatorname{Tr}\left(P_{a} \rho P_{a}\right)=\operatorname{Tr}\left(\rho P_{a}\right)$ is the probability assigned to the event $a$ by the state $\rho$.

If we consider a pair of correlated systems, $\mathrm{A}$ and $\mathrm{B}$, then conditionalization on an A-event, for the probabilistic information encoded in the density operator $\rho_{B}$ representing the probabilities of events at the remote system B, will always be an updating, in the sense of a refinement.

For example, suppose the system $\mathrm{A}$ is associated with a 3-dimensional Hilbert space $\mathcal{H}_{A}$ and the system $\mathrm{B}$ is associated with a 2-dimensional Hilbert space $\mathcal{H}_{B}$. Suppose the composite system $\mathrm{AB}$ is in an entangled state:

$$
\begin{aligned}
\left|\psi^{A B}\right\rangle & =\frac{1}{\sqrt{3}}\left(\left|a_{1}\right\rangle\left|b_{1}\right\rangle+\left|a_{2}\right\rangle|c\rangle+\left|a_{3}\right\rangle|d\rangle\right) \\
& =\frac{1}{\sqrt{3}}\left(\left|a_{1}^{\prime}\right\rangle\left|b_{2}\right\rangle+\left|a_{2}^{\prime}\right\rangle|e\rangle+\left|a_{3}^{\prime}\right\rangle|f\rangle\right)
\end{aligned}
$$

\footnotetext{
${ }^{5}$ See [9] for a discussion.
} 
where $\left|a_{1}\right\rangle,\left|a_{2}\right\rangle,\left|a_{3}\right\rangle$ and $\left|a_{1}^{\prime}\right\rangle,\left|a_{2}^{\prime}\right\rangle,\left|a_{3}^{\prime}\right\rangle$ are two orthonormal bases in $\mathcal{H}_{A}$ and $\left|b_{1}\right\rangle,\left|b_{2}\right\rangle$ is an orthonormal basis in $\mathcal{H}_{B}$. The triple $\left|b_{1}\right\rangle,|c\rangle,|d\rangle$ and the triple $\left|b_{2}\right\rangle,|e\rangle,|f\rangle$ are nonorthogonal triples of vectors in $\mathcal{H}_{B} .{ }^{6}$ The state of B (obtained by tracing over $\mathcal{H}_{A}$ ) is the completely mixed state $\rho_{B}=\frac{1}{2} I_{B}$ :

$$
\frac{1}{3}\left|b_{1}\right\rangle\left\langle b_{1}\left|+\frac{1}{3}\right| c\right\rangle\left\langle c\left|+\frac{1}{3}\right| d\right\rangle\left\langle d\left|=\frac{1}{3}\right| b_{2}\right\rangle\left\langle b_{2}\left|+\frac{1}{3}\right| e\right\rangle\left\langle e\left|+\frac{1}{3}\right| f\right\rangle\langle f|=\frac{I_{B}}{2}
$$

Conditionalizing on one of the eigenvalues $a_{1}, a_{2}, a_{3}$ or $a_{1}^{\prime}, a_{2}^{\prime}, a_{3}^{\prime}$ of an A-observable $A$ or $A^{\prime}$ via (4), i.e., on the occurrence of an event corresponding to $A$ taking the value $a_{i}$ or $A^{\prime}$ taking the value $a_{i}^{\prime}$ for some $i$, changes the density operator $\rho_{B}$ of the remote system $\mathrm{B}$ to one of the states $\left|b_{1}\right\rangle,|c\rangle,|d\rangle$ or to one of the states $\left|b_{2}\right\rangle,|e\rangle,|f\rangle$. Since the mixed state $\rho_{B}=\frac{1}{2} I_{B}$ can be decomposed as an equal weight mixture of $\left|b_{1}\right\rangle,|c\rangle,|d\rangle$ and as an equal weight mixture of $\left|b_{2}\right\rangle,|e\rangle,|f\rangle$, the change in the state of B is an updating, in the sense of a refinement of the information about $\mathrm{B}$ encoded in the state $\left|\psi^{A B}\right\rangle$, taking into account the new information $a_{i}$ or $a_{i}^{\prime}$. In fact, the mixed state $\rho_{B}=\frac{1}{2} I_{B}$ corresponds to an infinite variety of mixtures of pure states in $\mathcal{H}_{B}$ (not necessarily equal weight mixtures, of course). The effect at the remote system B of conditionalization on any event at A will always be an updating, in the sense of a refinement, with respect to one the these mixtures. ${ }^{7}$ This is the content of the Hughston-JozsaWootters theorem [23]. It is what Schrödinger called 'remote steering' and is the basis of quantum teleportation, quantum dense coding, and other peculiarities of quantum information, including the impossibility of unconditionally secure bit commitment (see Bub [10] for a discussion).

The effect of conditionalization at a remote system (the system that is not directly involved in the conditionalizing event) is then consistent with a 'no signaling' principle:

$$
\begin{aligned}
& \sum_{b} p(a b \mid A B) \equiv p(a \mid A B)=p(a \mid A) \\
& \sum_{a} p(a b \mid A B) \equiv p(b \mid A B)=p(b \mid B)
\end{aligned}
$$

where $a$ represents a value of $A$ and $b$ represents a value of $B$. If conditionalization on the value of an A-observable changed the probabilities at a remote system $\mathrm{B}$ in a way that could not be represented as an updating in the sense of a refinement of the prior information about $\mathrm{B}$ expressed in terms of correlations between A-observables and B-observables (as encoded in the entangled state $\left|\psi^{A B}\right\rangle$ ), then conditionalization would allow instantaneous signaling between $\mathrm{A}$ and $\mathrm{B}$. The occurrence of a particular event at $\mathrm{A}$-corresponding to a determinate value for the observable $A$ as opposed to a determinate value for $A^{\prime}$ —would produce a detectable change in the B-probabilities, and so Alice at A could signal instantaneously to Bob at B merely by performing an $A$ -measurement and gaining a specific sort of information about $\mathrm{A}$ (the value of $A$ or the value of $A^{\prime}$ ).

\footnotetext{
${ }^{6}$ The vectors in each triple are separated by an angle $2 \pi / 3$. For a precise specification of these vectors, see Bub [11]

${ }^{7}$ Fuchs makes a similar point in [15].
} 
The 'no signaling' principle is a special case of what Barnum et al [4] call 'the noncontextuality of probability,' which can be expressed as a condition on the probabilities assigned to the eigenvalues of any two commuting observables $[X, Y]=0$ :

$$
\begin{aligned}
& \sum_{y} p(x y \mid X Y) \equiv p(x \mid X Y) \quad=p(x \mid X) \\
& \sum_{x} p(x y \mid X Y) \equiv p(y \mid X Y) \quad=p(y \mid Y)
\end{aligned}
$$

This formulation of the noncontextuality of probability follows from the representation of an observable in terms of its spectral measure. ${ }^{8}$ We obtain the 'no signaling' condition if we take $X=A \otimes I$ and $Y=I \otimes B$. Note that 'no signaling' is not specifically a relativistic constraint on superluminal signaling. It is simply a condition imposed on the marginal probabilities of events for separated systems, requiring that the marginal probability of a B-event is independent of the particular set of mutually exclusive and collectively exhaustive events selected at A, and conversely, and this might well be considered partly constitutive of what one means by separated systems.

To preserve the 'no signaling' principle, quantum probabilities must also satisfy a 'no cloning' principle: there can be no universal cloning machine, i.e., it is impossible to construct a cloning machine that will clone the output of an arbitrary information source. More precisely, there can be no universal broadcasting machine-no device that takes a probability distribution over an event space to a new probability distribution over a product space of events, where the marginal probability distributions over each factor space is the same as the original distribution. We will continue to use the term 'cloning' rather than 'broadcasting' because it is more intuitive and more familiar, but note that we have in mind copying the outputs of an information source, not the information source itself (defined by the probability distribution).

Suppose a universal cloning machine were possible. Then such a device could copy any state in the orthogonal triple $\left|b_{1}\right\rangle,|c\rangle,|d\rangle$ as well as any state in the orthogonal triple $\left|b_{2}\right\rangle,|e\rangle,|f\rangle$. It would then be possible for Alice at A to signal to Bob at B. If Alice obtains the information given by an eigenvalue $a_{i}$ of $A$ or $a_{i}^{\prime}$ of $A^{\prime}$, and Bob inputs the system $\mathrm{B}$ into the cloning device $n$ times, he will obtain one of the states $\left|b_{1}\right\rangle^{\otimes n},|c\rangle^{\otimes n},|d\rangle^{\otimes n}$ or one of the states $\left|b_{2}\right\rangle^{\otimes n},|e\rangle^{\otimes n},|f\rangle^{\otimes n}$, depending on the nature of Alice's information. Since these states tend to mutual orthogonality in $\otimes^{n} \mathcal{H}_{\mathcal{B}}$ as $n \rightarrow \infty$, they are distinguishable in the limit. So, even for finite $n$, Bob would in principle be able to obtain some information instantaneously about a remote event.

More fundamentally, the existence of a universal cloning machine is inconsistent with the interpretation of Hilbert space as providing the kinematic framework for an indeterministic physics, in which probabilities (objective chances) are 'uniquely given from the start' by the geometry of Hilbert space. For such a device would be able to distinguish the equivalent mixtures of nonorthogonal states represented by the same density operator $\rho_{B}=\frac{1}{2} I_{B}$. If a quantum state prepared as an equal weight mixture of

\footnotetext{
${ }^{8}$ Barnum et al formulate noncontextuality as the requirement that the probability assigned to an event $e$ depends only on $e$ and is independent of the other events in each mutually exclusive and collectively exhaustive set of events $\left\{e_{i}\right\}$ containing $e$, i.e., that the probability of an event is independent of the Boolean subalgebra to which the event belongs.
} 
the states $\left|b_{1}\right\rangle,|c\rangle,|d\rangle$ could be distinguished from a state prepared as an equal weight mixture of the states $\left|b_{2}\right\rangle,|e\rangle,|f\rangle$, the representation of quantum states by density operators would be incomplete.

Now consider the effect of conditionalization on the state of $A$. The state of $A B$ can be expressed as the biorthogonal (Schmidt) decomposition:

$$
\left|\psi^{A B}\right\rangle=\frac{1}{\sqrt{2}}\left(|g\rangle\left|b_{1}\right\rangle+|h\rangle\left|b_{2}\right\rangle\right)
$$

where

$$
\begin{aligned}
|g\rangle & =\frac{2\left|a_{1}\right\rangle-\left|a_{2}\right\rangle-\left|a_{3}\right\rangle}{\sqrt{6}} \\
|h\rangle & =\frac{\left|a_{2}\right\rangle-\left|a_{3}\right\rangle}{\sqrt{2}}
\end{aligned}
$$

The density operator $\rho_{A}$, obtained by tracing $\left|\psi^{A B}\right\rangle$ over $\mathrm{B}$, is:

$$
\rho_{A}=\frac{1}{2}|g\rangle\left\langle g\left|+\frac{1}{2}\right| h\right\rangle\langle h|
$$

which has support on a 2-dimensional subspace in the 3-dimensional Hilbert space $\mathcal{H}_{A}$ : the plane spanned by $|g\rangle$ and $|h\rangle$ (in fact, $\rho_{A}=\frac{1}{2} P_{A}$, where $P_{A}$ is the projection operator onto the plane). Conditionalizing on a value of $A$ or $A^{\prime}$ yields a state that has a component outside this plane. So the state change on conditionalization cannot be interpreted as an updating of information in the sense of a refinement, i.e., as the selection of a particular alternative among a set of mutually exclusive and collectively exhaustive alternatives represented by the state $\rho_{A}$.

This is the notorious 'irreducible and uncontrollable disturbance' arising in the registration of new information about the occurrence of an event that underlies the measurement problem: the loss of some of the information encoded in the original state (in the above example, the probability of the A-event represented by the projection operator onto the 2-dimensional subspace $P_{A}$ is no longer 1, after the registration of the new information about the observable $A$ or $A^{\prime}$ ). If the registration of new information is the outcome of a measurement then, since the state change on measurement will have to be stochastic and non-unitary, it cannot be described by the deterministic dynamics of the theory, which must be unitary (for closed systems) for consistency with the Hilbert space representation of probabilities. A solution to the problem is generally understood to require amending the theory in such a way that the loss of information can be accounted for dynamically, and the quantum probabilities can be reconstructed dynamically as measurement probabilities. Then the quantum probabilities are not 'uniquely given from the start' as kinematic features of an appropriately represented event structure, i.e., they do not arise kinematically but are derived dynamically, as artifacts of the measurement process or of decoherence. Even on the Everett interpretation, where Hilbert space is interpreted as the representation space for a new sort of ontological entity, represented by the quantum state, and no definite outcome out of a range of alternative outcomes is selected in a quantum measurement process (so no explanation 
is required for such an event), probabilities arise as a feature of the branching structure that emerges in the dynamical process of decoherence.

From the perspective of the information-theoretic interpretation, the 'disturbance' involved in conditionalization is a kinematic phenomenon associated with the nonBoolean quantum event space. If there were no information loss in the conditionalization of quantum probabilities, then cloning would be possible, and equivalent mixtures associated with the same density operator would be distinguishable, in which case Hilbert space would not be an appropriate representation space for quantum events and their probabilistic correlations. ${ }^{9}$ In the Appendix, we show that this follows directly from the 'no cloning' principle for a large class of theories. We prove that in this class of theories the 'no cloning' principle demarcates the boundary between classical theories and theories in which measurement involves an 'irreducible and uncontrollable disturbance'. It seems plausible, therefore, that this principle should play a central role in a derivation of the Hilbert space structure from information theory.

It is instructive here to recall Einstein's distinction between 'principle' theories, like the special theory of relativity, formulated in terms of the relativity principle and the light postulate (empirical regularities raised to the level of postulates), and 'constructive' theories, like Lorentz's theory, formulated in terms of a rich ontology of objects like particles, fields, and the ether. Einstein compared thermodynamics as a principle theory ('no perpetual motion machines of the first and and second kind') to the kinetic theory of gases as a constructive theory (where the mechanical and thermal behavior of a gas is reduced to the motion of molecules, modeled as little billiard balls). He proposed special relativity as a kinematic replacement for Lorentz's dynamical interpretation of what we now refer to as Lorentz invariance, which he saw as unsatisfactory, not as a rival theory of matter and radiation. One might say that what eventually replaced Lorentz's theory was relativistic quantum theory. From this perspective, Minkowski space-time is the constructive theory corresponding to Einstein's principle theory formulation of special relativity: it is a component of the kinematic part of the constructive theory of the constitution of matter provided by relativistic quantum theory. (See Janssen [1, 331-332] for an account along these lines.)

In an article entitled 'How to Teach Special Relativity' [5], John Bell considers the following puzzle: Three identical spaceships, $A, B$, and $C$, are at rest relative to one other, drifting freely far from other matter without rotation, with $A$ equidistant from $B$ and $C$. The spaceships $B$ and $C$ are connected by a fragile thread, which is just long enough to span the distance between them. On reception of a signal from $A$, the spaceships $B$ and $C$ start their engines and accelerate gently. Since $B$ and $C$ are assumed to be identical, with identical acceleration programs, they will have the same velocity and so remain separated by the same distance relative to $A$. When $B$ and $C$ reach a certain velocity, the thread breaks. The question is: why does the thread break? Note that the thread would not break in a Newtonian universe.

The relativistic kinematical explanation goes along the following lines:

Let $F 1$ be the inertial frame in which the spaceships $A, B, C$ are initially at rest (and $A$ remains at rest). In $F 1$, the distance between $B$ and $C$, as the spaceships begin

\footnotetext{
${ }^{9}$ For the Everettian, there is the appearance of measurement disturbance on each branch, or rather, on 'most' branches, because there will always be some branches on which it appears that there is no measurement disturbance - and on these branches it will appear that cloning is possible.
} 
to move and continue moving, remains the same as the initial resting distance. But the moving thread undergoes a Lorentz contraction in the direction of its motion in $F 1$. The explanation, in $F 1$, of why the thread breaks is just this: the thread breaks because it is contracting, and this contraction is resisted by the thread being tied to $B$ and $C$, which maintain a distance apart greater than the contraction requires. The thread will break when $B$ and $C$ reach a sufficiently high velocity in $F 1$ and the prevention of the Lorentz contraction produces sufficient stress to break the thread.

Let $F 2$ be the inertial frame in which $B$ and $C$ are finally at rest again, after their engines have been shut off. From the perspective of $F 2$, there is a different explanation for the thread breaking. In $F 2$, the two spaceships $B$ and $C$ are decelerating, and eventually come to rest. However, they are not decelerating at the same rate (they would be if $B$ and $C$ were connected by a rigid rod). It is this difference in deceleration that is responsible for the stress in the thread, which eventually causes the thread to break.

To clarify further, one might consider two additional spaceships, $E$ and $F$, identical to $B$ and $C$, with identical acceleration programs, initially at rest in $F 1$ (before $B$ and $C$ start their engines), with $E$ adjacent to $B$, and $F$ adjacent to $C$. Suppose $E$ and $F$ are connected rigidly, so that $E F$ behaves like a rigid rod with the two spaceships as endpoints, initially at rest in $F 1$. Suppose also that $E F$ starts accelerating at the same time as $B$ and $C$ in $F 1$, and that the rod connecting $E$ and $F$ is strong enough to remain rigid under the acceleration. Bell's characterization of the setup requires that, in $F 1$, the distance between $B$ and $C$, as the spaceships begin to move and continue moving, remains the same as the initial resting distance. So, in $F 1$, this distance will become greater than the distance between $E$ and $F$, once the spaceships start moving, since $E F$ will suffer a Lorentz contraction in the direction of its motion. In the explanation in frame $F 1$, the thread breaks because it is contracting by as much as $E F$ contracts. In the explanation in frame $F 2, B$ and $C$ are not decelerating at the same rate-rather, the endpoints of $E F$ are decelerating at the same rate-and this difference in deceleration, relative to the deceleration of $E F$, is responsible for the stress in the thread, which eventually causes it to break.

The explanations are frame-dependent, insofar as they involve elements that are frame-dependent notions in special relativity. However, the increasing stress in the thread that causes it to break, and the fact that the thread breaks when the stress exceeds the tensile strength of the thread, are frame-independent features common to all explanations. What Bell pointed out was that one ought to be able to provide an explanation for the thread breaking in terms of an explicit calculation of the forces involved, and the tensile strength of the thread. He suggests that such a dynamical explanation is a deeper or at least more informative explanation than the kinematic explanation. Harvey Brown's book Physical Relativity [7] develops this theme.

In Bell's spaceship example, the dynamical explanation for the thread breaking in terms of forces, insofar as the forces are Lorentz invariant, shows the possibility of a dynamics consistent with the kinematics of special relativity. The only factor relevant to the thread breaking is the Lorentz contraction, a feature of the geometry of Minkowski space-time which is quite independent of the material constitution of the thread and the nature of the specific interactions involved. Given Einstein's two principles, there is no deeper explanation for the thread breaking than the kinematical expla- 
nation provided by the structure of Minkowski space-time. ${ }^{10}$ The demonstration that a dynamical explanation yields the same result as the kinematical explanation sketched above amounts to a consistency proof that a relativistic dynamics - a dynamics that conforms to the structure of Minkowski space-time-is possible.

If we take special relativity as a template for the analysis of quantum conditionalization and the associated measurement problem, ${ }^{11}$ the information-theoretic view of quantum probabilities as 'uniquely given from the start' by the structure of Hilbert space as a kinematic framework for an indeterministic physics is the proposal to interpret Hilbert space as a constructive theory of information-theoretic structure or probabilistic structure, part of the kinematics of a full constructive theory of the constitution of matter, where the corresponding principle theory includes information-theoretic constraints such as 'no signaling' and 'no cloning.' ${ }^{12}$ Lorentz contraction is a physically real phenomenon explained relativistically as a kinematic effect of motion in a non-Newtonian space-time structure. Analogously, the change arising in quantum conditionalization that involves a real loss of information is explained quantum mechanically as a kinematic effect of any process of gaining information of the relevant sort in the non-Boolean probability structure of Hilbert space (irrespective of the dynamical processes involved in the measurement process). Given 'no cloning' as a fundamental principle, there can be no deeper explanation for the information loss on conditionalization than that provided by the structure of Hilbert space as a probability theory or information theory. The definite occurrence of a particular event is constrained by the kinematic probabilistic correlations encoded in the structure of Hilbert space, and only by these correlations-it is otherwise 'free.'

The Born weights are probabilities in a purely formal sense unless they are related to experience by some explicitly formulated principle. The cash value of the 'transition probability' $|\langle e \mid f\rangle|^{2}$ is that $|\langle e \mid f\rangle|^{2}$ represents the probability, in the state $|e\rangle$, of finding the outcome corresponding to the state $|f\rangle$ in a measurement of an observable of which $|f\rangle$ is an eigenstate. But if quantum mechanics is more than an instrument for predicting the probabilities of measurement outcomes, it must be possible, in principle, to locate structures that represent macroscopic measuring instruments and recording devices in Hilbert space, where the dynamical behavior of such structures is consistent with the kinematic information-theoretic (probabilistic) principles encoded in the structure of Hilbert space.

In special relativity one has a consistency proof that a dynamical account of relativistic phenomena in terms of forces, like the breaking of the thread in Bell's spaceship example, is consistent with the kinematical account in terms of the structure of Minkowski space-time. An analogous consistency proof for quantum mechanics would be a dynamical explanation for the effective emergence of classicality, i.e., Booleanity, at the macrolevel, because it is with respect to the Boolean algebra of the macroworld that the Born weights of quantum mechanics have empirical cash value.

\footnotetext{
${ }^{10}$ Harvey Brown's book [7] presents an extended argument for the contrary view.

${ }^{11}$ See Brown and Timpson [8] for a contrary view.

${ }^{12}$ While the 'no cloning' principle demarcates classical from non-classical theories, we require some further principle or principles to recover Hilbert space and exclude 'superquantum' theories for which the correlation of entangled states violates the Tsirelson bound for quantum states, while conforming to the "no signaling' constraint. See Barnum et al [2,3].
} 
In classical mechanics, taking a Laplacian view, one can consider the phase space of the entire universe, in principle. The classical state, represented by a point in phase space that evolves dynamically, defines a 2-valued homomorphism on the Boolean algebra of (Borel) subsets of phase space, distinguishing events that occur at a particular time from events that don't occur. In this sense, the classical state is the 'truthmaker' for propositions about the occurrence or non-occurrenc of events, for all possible events.

Similarly, in quantum mechanics one can consider the Hilbert space of the entire universe, in principle. This is a space of possible events, with a certain kinematic structure of probabilistic correlations between events, represented by the subspace structure or projective geometry of the space (different from the classical correlational structure represented by the subset structure of phase space). On the usual view, the quantum analogue of the classical state is a pure state represented by a ray or 1-dimensional subspace in Hilbert space. There is, of course, no 2-valued homomorphism on the non-Boolean algebra of subspaces of Hilbert space, but a pure state can be taken as distinguishing events that occur at a particular time (events represented by subspaces containing the state, and assigned probability 1 by the state) from events that don't occur (events represented by subspaces orthogonal to the state, and assigned probability 0 by the state). This leaves all remaining events represented by subspaces that neither contain the state nor are orthogonal to the state (i.e., events assigned a probability $p$ by the state, where $0<p<1$ ) in limbo: neither occurring nor not occurring. The measurement problem then arises as the problem of accounting for the fact that an event that neither occurs not does not occur when the system is in a given quantum state can somehow occur when the system undergoes a measurement interaction with a macroscopic measurement device_-giving measurement a very special status in the theory. Once the pure state is taken as the analogue of the classical state in this sense, the only way out of this problem, without adding structure to the theory, is the Everettian manoeuvre.

On the information-theoretic interpretation, the quantum state is a derived entity, a credence function that assigns probabilities to events in alternative Boolean algebras associated with the outcomes of alternative measurement outcomes. The measurement outcomes are macro-events in a particular Boolean algebra, and the macro-events that actually occur, corresponding to a particular measurement outcome, define a 2-valued homomorphism on this Boolean algebra. What has to be shown is how this occurrence of events in a particular Boolean algebra is consistent with the quantum dynamics.

It is a contingent feature of the dynamics of our quantum universe that events represented by subspaces of Hilbert space have a tensor product structure that reflects the division of the universe into microsystems (e.g., atomic nuclei), macrosystems (e.g., macroscopic measurement devices constructed from pieces of metal and other hardware), and the environment (e.g., air molecules, electromagnetic radiation). The Hamiltonians characterizing the interactions between microsystems and macrosystems, and the interactions between macrosystems and their environment, are such that a certain relative structural stability emerges at the macrolevel. The unitary dynamics (in the Heisenberg picture) evolves the entire tensor-product structure of events in Hilbert space. Symbolically, events like

$$
|s\rangle|M\rangle|\varepsilon\rangle
$$


where $s, M, \varepsilon$ represent respectively microsystem, macrosystem, and environment, evolve to

$$
\sum_{k} c_{k}\left|s_{k}\right\rangle\left|M_{k}\right\rangle\left|\varepsilon_{k}(t)\right\rangle
$$

if the interaction Hamiltonian $H_{M \varepsilon}$ between a macrosystem and the environment is of the form

$$
H_{M \varepsilon}=\sum_{k \gamma} g_{k \nu}\left|M_{k}\right\rangle\left\langle M_{k}|\otimes| e_{\nu}\right\rangle\left\langle e_{\nu}\right|
$$

where the $\left|M_{k}\right\rangle$ and the $\left|e_{k}\right\rangle$ are orthogonal. That is, the 'pointer' observable $\sum_{k} m_{k}\left|M_{k}\right\rangle\left\langle M_{k}\right|$ commutes with $H_{M \varepsilon}$, so the 'pointer' observable is a constant of the motion induced by the Hamiltonian $H_{M \varepsilon}$. Here, taking a 'Laplacian' view, $\left|M_{k}\right\rangle$ can be taken as representing, in principle, a configuration of the entire macroworld, and $\left|s_{k}\right\rangle$ a configuration of all the micro-events correlated with macro-events. The dynamics preserves the correlation $\sum_{k} c_{k}\left|s_{k}\right\rangle\left|M_{k}\right\rangle\left|\varepsilon_{k}(t)\right\rangle$ between micro-events, macro-events, and the environment for the macro-events $\left|M_{k}\right\rangle$, even for nonorthogonal $\left|s_{k}\right\rangle$ and $\left|\varepsilon_{k}\right\rangle$, but not for macro-events $\left|M_{l}^{\prime}\right\rangle$ that are linear superpositions of the $\left|M_{k}\right\rangle$. Since the tri-decomposition $\sum_{k} c_{k}\left|s_{k}\right\rangle\left|M_{k}\right\rangle\left|\varepsilon_{k}(t)\right\rangle$ is unique (unlike the biorthogonal Schmidt decomposition; see Elby and Bub [14]), a correlation of the form $|s\rangle|M\rangle|\varepsilon\rangle$ evolves to a linear superposition in which the macro-events $\left|M_{l}^{\prime}\right\rangle$ become correlated with entangled system-environment events represented by linear superpositions of the form $\sum_{k} c_{k} d_{l k}\left|s_{k}\right\rangle\left|\varepsilon_{k}(t)\right\rangle$. (See Zurek [39, p. 052105-14].)

It is characteristic of the dynamics that correlations represented by (16) evolve to similar correlations, and the macro-events represented by $\left|M_{k}\right\rangle$, at a sufficient level of coarse-graining, can be associated with structures at the macrolevel-the familiar macro-objects of our experience- that remain relatively stable under the dynamical evolution. So a Boolean algebra $\mathcal{B}_{\mathcal{M}}$ of macro-events $\left|M_{k}\right\rangle$ correlated with microevents $\left|s_{k}\right\rangle$ in (16) is emergent in the dynamics. Note that the emergent Boolean algebra is not the same Boolean algebra from moment to moment, because the correlation between micro-events and macro-events changes under the dynamical evolution induced by the micro-macro interaction (e.g., corresponding to different measurement interactions). What remains relatively stable under the dynamical evolution are the macrosystems associated with macro-events in correlations of the form (16), even under a certain vagueness in the coarse-graining associated with these macro-events: macrosystems like grains of sand, tables and chairs, macroscopic measurement devices, cats and people, galaxies, etc.

It is further characteristic of the dynamics that the environmental events represented by

$$
\left|\varepsilon_{k}(t)\right\rangle=\sum_{\nu} e^{-i g_{k \nu} t}\left|e_{\nu}\right\rangle
$$

very rapidly approach orthogonality, i.e., the 'decoherence factor'

$$
\zeta_{k k^{\prime}}=\left\langle\varepsilon_{k} \mid \varepsilon_{k^{\prime}}\right\rangle=\sum_{\nu}\left|\gamma_{\nu}\right|^{2} e^{i\left(g_{k^{\prime} \nu}-g_{k \nu}\right) t}
$$

becomes negligibly small almost instantaneously. When the environmental events $\left|\varepsilon_{k}(t)\right\rangle$ correlated with the macro-events $\left|M_{k}\right\rangle$ are effectively orthogonal, the reduced 
density operator is effectively diagonal in the 'pointer' basis $\left|M_{k}\right\rangle$ and there is effectively no interference between elements of the emergent Boolean algebra $\mathcal{B}_{\mathcal{M}}$, i.e., the conditional probabilities of events associated with a subsequent emergent Boolean algebra (a subsequent measurement) is additive on $\mathcal{B}_{\mathcal{M}}$. (See Zurek [39, p. 052105-14], [38].)

From a 'Laplacian' point of view, the Born probabilities are probabilities of events in the emergent Boolean algebra, i.e., the Born probabilities are probabilities of 'pointer' positions, the coarse-grained basis selected by the dynamics. Applying quantum mechanics kinematically, say in assigning probabilities to the possible outcomes of a measurement of some observable of a microsystem, we consider the Hilbert space of the relevant degrees of freedom of the microsystem and treat the measuring instrument as simply selecting a Boolean subalgebra in the non-Boolean event space of the microsystem to which the Born probabilities apply. In principle, we can include the measuring instrument in a dynamical analysis of the measurement process, but such a dynamical analysis - even though complete in terms of the quantum dynamics-does not provide a dynamical explanation of how individual outcomes come about. In such a dynamical analysis, the Born probabilities are probabilities of the occurrence of events in an emergent Boolean algebra. The information loss on conditionalization relative to classical conditionalization is a kinematic feature of the the structure of quantum events, not accounted for by the unitary quantum dynamics, which conforms to the kinematic structure. This is analogous to the situation in special relativity, where Lorentz contraction is a kinematic effect of relative motion that is consistent with a dynamical account in terms of Lorentz covariant forces, but is not explained in Einstein's theory-by contrast with Lorentz's theory-as a dynamical effect in a Newtonian space-time structure, in which this sort of contraction does not arise as a purely kinematic effect. That is, the dynamical explanation of Lorentz contraction in special relativity involves forces that are Lorentz invariant-in effect, the dynamics is assumed to have symmetries that respect Lorentz contraction as a kinematical effect of relative motion. In quantum mechanics, the possibility of a dynamical analysis of the measurement process conforming to the kinematic structure of Hilbert space provides a consistency proof that the familiar objects of our macroworld behave dynamically in accordance with the kinematic probabilistic constraints on correlations between events.

A physical theory of an indeterministic universe is primarily a theory of probability (or information). Probabilities are defined over an event structure, which in the quantum case is a family of Boolean algebras forming a particular sort of non-Boolean algebra. On the information-theoretic interpretation, no assumption is made about the fundamental 'stuff' of the universe. So, one might ask, what do tigers supervene on? ${ }^{13}$ In the case of Bohm's theory or the GRW theory, the answer is relatively straightforward: tigers supervene on particle configurations in the case of Bohm's theory, and on (collapsed) wavefunctions in the case of the GRW theory. In the Everett interpretation, tigers supervene on features of the quantum state, which describes an ontological entity. In the case of the information-theoretic interpretation, the 'supervenience base' is provided by the dynamical analysis: tigers supervene on events defining a 2-valued homomorphism in the emergent Boolean algebra.

\footnotetext{
${ }^{13}$ We thank Allen Stairs for raising the realism question in this form.
} 
It might be supposed that this involves a contradiction. What is contradictory, given our assumptions about the kinematic significance of the Hilbert space, is to suppose that a correlational event represented by a 1-dimensional subspace like $\sum_{k} c_{k}\left|s_{k}\right\rangle\left|M_{k}\right\rangle\left|\varepsilon_{k}(t)\right\rangle$ actually occurs, as well as an event represented by a 1-dimensional subspace like $\left|s_{k}\right\rangle\left|M_{k}\right\rangle\left|\varepsilon_{k}(t)\right\rangle$ for some specific $k$. We do not suppose this. On the informationtheoretic interpretation we propose, there is a kinematic structure of possible correlations (but no particular atomic correlational event is selected as the 'state' in a sense analogous to the classical state), and a particular dynamics that preserves certain sorts of correlations, i.e., correlational events of the sort represented by $\sum_{k} c_{k}\left|s_{k}\right\rangle\left|M_{k}\right\rangle\left|\varepsilon_{k}(t)\right\rangle$ evolve to correlational events of the same form. What can be identified as emergent in this dynamics is an effectively classical probability space: a Boolean algebra with atomic correlational events of the sort represented by orthogonal 1-dimensional subspaces $\left|s_{k}\right\rangle\left|M_{k}\right\rangle$, where the probabilities are generated by the reduced density operator obtained by tracing over the environment, when the correlated environmental events are effectively orthogonal.

Since this is an indeterministic theory, the dynamics does not describe the (deterministic or stochastic) evolution of the 2-valued homomorphism on which tigers supervene to a new 2-valued homomorphism (as in the evolution of a classical state). Rather, the dynamics leads to the relative stability of certain event structures at the macrolevel associated with the familiar macrosystems of our experience, and to an emergent effectively classical probability space whose atomic events are correlations between events associated with these macrosystems and micro-events. It is part of the information-theoretic interpretation that events defining a 2-valued homomorphism on the Boolean algebra of this classical probability space actually occur with the emergence of the Boolean algebra at the macrolevel. This selection of actually occurring events is only in conflict with the quantum state if the quantum state is assumed to have an ontological significance analogous to the ontological significance of the classical state as the 'truthmaker' for propositions about the occurrence and non-occurrence of events, and if the quantum state evolves unitarily-in particular, if it is assumed that the quantum state partitions all events into events that actually occur, events that do not occur, and events that neither occur nor do not occur, as on the usual interpretation. We argued that this assumption is one of the dogmas about quantum mechanics that should be rejected. Rather, we take the quantum state to be a credence function: the credence function of a rational agent (an information-gathering entity 'in' the emergent Boolean algebra) who is updating probabilities on the basis of events that-from a 'Laplacian' point of view-occur in the emergent Boolean algebra.

\section{Concluding Remarks}

The common assumption of the Everett interpretation and the GRW theory is that the quantum state has an ontological significance analogous to the ontological significance of the classical state as the 'truthmaker' for propositions about the occurrence and non-occurrence of events - that the quantum state describes what there is at the most fundamental level. In the case of Bohm's theory, the quantum state is part of the story, the guiding field for the particle trajectories, and the particle configuration is the "truth- 
maker.' Once this assumption is accepted, the measurement problem arises as the problem of explaining how it makes sense to take a pure quantum state decomposed into a superposition as representing what there is, while at the same time interpreting the state as assigning probabilities to the states in the superposition, if this is understood to mean that these pure states describe alternative complexes of events, only one of which actually occurs with the associated probability (since the occurrence of the event complex represented by the superposition conflicts with the occurrence of each of the event complexes represented by the pure states in the superposition). If the probabilities are interpreted as the probabilities of alternative possible measurement outcomes, when a measurement is performed whose possible outcomes are represented by the pure states in the superposition, i.e., as the probabilities that the quantum state will undergo alternative possible dynamical transitions to new states compatible with the alternative possible measurement outcomes, then it seems that we are required to provide a dynamical account of how the actual outcome comes about in a measurement process when the appropriate measurement interaction is implemented, as opposed to other possible outcomes, unless we are content with an instrumentalist interpretation of quantum mechanics as an algorithm for the probabilities of measurement outcomes. But such a dynamical account is impossible if measurement is modeled as an interaction that conforms to the dynamics of the theory and no further element of structure is added to the theory.

We have argued that the 'big' measurement problem, which is at issues here, is like the problem for Newtonian physics raised by relativistic effects such as length contraction and time dilation, and that the solution to both problems involves the recognition of a fundamental change in the underlying kinematics of our physics, represented by the transition from a Newtonian space-time to Minkowski space-time in the case of special relativity, and from the set-theoretic structure of classical phase space to the subspace structure of Hilbert space in the case of quantum mechanics. So the two assumptions, about the ontological significance of the quantum state and about the dynamical account of how measurement outcomes come about, should be rejected as unwarranted dogmas about quantum mechanics.

The solutions to the 'big' measurement problem provided by Bohm's theory and the GRW theory are dynamical and involve adding structure to quantum mechanics. There is a sense in which adding structure to the theory to solve the measurement problem dynamically_insofar as the problem arises from a failure to recognize the significance of Hilbert space as the kinematic framework for the physics of an indeterministic universe-is like Lorentz's attempt to explain relativistic length contraction dynamically, taking the Newtonian space-time structure as the underlying kinematics and invoking the ether as an additional structure for the propagation of electromagnetic effects. In this sense, Bohm's theory and the GRW theory are 'Lorentzian' interpretations of quantum mechanics.

The Everettian rejects the legitimacy of the problem by simply denying that measurements have definite outcomes, i.e., by denying that the pure states in a superposition describe alternative event complexes, only one of which actually occurs. This requires showing that a particular decomposition of the quantum state corresponding to our experience has a preferred significance, and that weights can be assigned to the individual terms in the preferred superposition that have the significance of probabil- 
ities, even though no one definite event complex is selected as actually occurring in contrast to the other event complexes in the superposition. The Everettian's solution to this problem is dynamical. So the Everettian, too, sees the underlying problem as dynamical.

We reject the legitimacy of the 'big' measurement problem on the basis of an information-theoretic interpretation of quantum mechanics, in terms of which the problem arises from the failure to see the significance of Hilbert space as the kinematic framework for an indeterministic physics. The dynamical analysis we provide is a solution to a consistency problem: the 'small' measurement problem. The analysis shows that a quantum dynamics, consistent with the kinematics of Hilbert space, suffices to underwrite the emergence of a classical probability space for the familiar macro-events of our experience, with the Born probabilities for macro-events associated with measurement outcomes derived from the quantum state as a credence function. The explanation for such nonclassical effects as the loss of information on conditionalization is not provided by the dynamics, but by the kinematics, and given 'no cloning' as a fundamental principle, there can be no deeper explanation. In particular, there is no dynamical explanation for the definite occurrence of a particular measurement outcome, as opposed to other possible measurement outcomes in a quantum measurement process - the occurrence is constrained by the kinematic probabilistic correlations encoded in the projective geometry of Hilbert space, and only by these correlations.

\section{Appendix: The Information Loss Theorem}

We show that it follows from the 'no cloning' principle that information cannot be extracted from a nonclassical source without changing the source irreversibly. (We prove this theorem for quantum information sources, but note that the proof does not depend on specific features of the Hilbert space formalism.)

We assume:

(1) The 'no cloning' principle: there is no universal cloning machine.

(2) Every (quantum) state $\rho$ is specified by the probabilities of the measurement outcomes of a finite, informationally complete (or 'fiducial') set of observables.

Assumption (2) holds for a large class of theories, including quantum and classical theories. Note that an informationally complete set is not unique. For example, in the case of a qubit, the probabilities for spin 'up' and spin 'down' in three orthogonal directions suffice to define a direction on the Bloch sphere and hence to determine the state, so the spin observables $\sigma_{x}, \sigma_{y}, \sigma_{z}$ form an informationally complete set. (For a classical system or a classical information source, an informationally complete set is given by of a single observable, with $n$ possible outcomes, for some $n$.)

Let $\mathcal{F}=\{A, B, C, \ldots\}$ be an informationally complete set of observables represented by a finite set of Hermitian operators on an $n$-dimensional Hilbert space $\mathcal{H}_{n}$. A quantum state $\rho$ assigns a probability distribution to every outcome of any measurement of an obervable in $\mathcal{F}$. Measuring $A$ yields one of the outcomes $a_{1}, a_{2}, \ldots$ with a probability distribution $P_{\rho}\left(a_{1} \mid A\right), P_{\rho}\left(a_{2} \mid A\right), \ldots$ Similarly, measuring $B$ yields 
one of the outcomes $b_{1}, b_{2}, \ldots$ with a probability distribution $P_{\rho}\left(b_{1} \mid A\right), P_{\rho}\left(b_{2} \mid A\right), \ldots$, and so on. If $\mathcal{F}$ is informationally complete, the finite set of probabilities completely characterizes $\rho$ as the state on $\mathcal{H}$.

Assuming that all measurement outcomes are independent and ignoring any algebraic relations among elements of $\mathcal{F}$, a classical probability measure on a classical (Kolmogorov) probability space can be constructed from these probabilities:

$$
P_{\rho}(a, b, \ldots \mid A, B, \ldots)=P_{\rho}(a \mid A) P_{\rho}(b \mid B) \ldots
$$

(cf. the 'trivial' hidden variable construction of Kochen and Specker in [25]). Note that the probability space is finite since $\mathcal{F}$ is finite and $\operatorname{dim} \mathcal{H}<\infty$. (The number of atoms in the probability space is at most $\operatorname{dim} \mathcal{H}^{|\mathcal{F}|}$.) The quantum state $\rho$ can be reconstructed from $P_{\rho}$ (given as a classical information source, or rationally approximated in the memory of a classical computer).

We now prove:

The Information Loss Theorem. Assumptions (1) and (2) entail that extracting information from a quantum information source given by a quantum state $\rho$, sufficient to generate the probabilities of an informationally complete set of observables, is either impossible or necessarily changes the state $\rho$ irreversibly, i.e., there must be information loss in the extraction of such information.

Proof. Step 1: begin with a quantum source in the state $\rho$ and measure $A, B, \ldots$ sufficiently many times to generate the classical probability measure $P_{\rho}$, to as good an approximation as required, without destroying $\rho$. Step 2: from $P_{\rho}$ construct a copy of $\rho$.

$$
\rho \stackrel{\text { measure }}{\longrightarrow} P_{\rho} \stackrel{\text { prepare }}{\longrightarrow} \rho
$$

This procedure defines a universal cloning machine, which we assume to be impossible. Since Step 2 is possible by assumption (2), the 'no cloning' assumption (1) entails that Step 1 is blocked.

We are left with two options: either there is no way to generate $P_{\rho}$ from $\rho$ (which is the case in quantum mechanics if we have only one copy of $\rho$, or too few copies of $\rho$ ), or else, if we can generate $P_{\rho}$ from $\rho$, assumption (1) entails that the original 'blueprint' $\rho$ must have been changed irreversibly by the process of extracting the information to generate $P_{\rho}$ (if not, the change in $\rho$ could be reversed dynamically and cloning would be possible):

$$
\not k \stackrel{\text { measure }}{\longrightarrow} P_{\rho} \stackrel{\text { prepare }}{\longrightarrow} \rho
$$

Since we can prepare multiple copies of the state $\rho$ from $P_{\rho}$, one might think that even if the original state is destroyed in generating $P_{\rho}$, we still end up with multiple copies of $\rho$ :

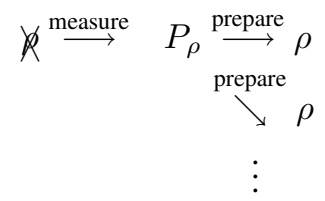


But note that to generate $P_{\rho}$, we need to begin with multiple copies of $\rho$, i.e., we need to begin with a state $\rho \otimes \rho \cdots$, so what we really have is:

$$
\not k \otimes \not K \cdots \stackrel{\text { measure }}{\longrightarrow} P_{\rho} \stackrel{\text { prepare }}{\longrightarrow} \rho \otimes \rho \cdots
$$

which simply re-states (22).

Corollary. No complete dynamical (i.e., unitary) account of the state transition in a measurement process is possible in quantum mechanics, in general.

Proof. Any measurement can be part of an informationally complete set, so any measurement must lead to an irreversible (hence non-unitary) change in the quantum state of the measured system.

We conclude-essentially from the 'no cloning' principle-that there can be no measurement device that functions dynamically in such a way as to identify with certainty the output of an arbitrary quantum information source without altering the source irreversibly or 'uncontrollably,' to use Bohr's term—no device can distinguish a given output from every other possible output by undergoing a dynamical (unitary) transformation that results in a state that represents a distinguishable record of the output, without an irreversible transformation of the source.

\section{References}

[1] Yuri Balashov and Michel Janssen. Critical notice: Presentism and relativity. British Journal for the Philosophy of Science, 54:327-346, 2003.

[2] Howard Barnum, Jonathan Barrett, Matthew Leifer, and Alexander Wilce. Cloning and broadcasting in generic probabilistic models. quant-ph/0611295, 2006.

[3] Howard Barnum, Jonathan Barrett, Matthew Leifer, and Alexander Wilce. A generalized no-broadcasting theorem. quant-ph/0707.0620, 2007.

[4] Howard Barnum, Carlton M. Caves, David Finkestein, Christopher A. Fuchs, and Rüdiger Schack. Quantum probability from decision theory? Proceedings of the Royal Society of London, A456:1175-1182, 2000.

[5] John Stuart Bell. How to teach special relativity. In Speakable and Unspeakable in Quantum Mechanics, pages 67-80. Cambridge University Press, Cambridge, 1987.

[6] John Stuart Bell. Against measurement. Physics World, 8:33-40, 1990. Reprinted in Sixty-Two Years of Uncertainty: Historical, Philosophical and Physical Inquiries into the Foundations of Quantum Mechanics, Arthur Miller (ed.), pp. 1731, Plenum, New York (1990).

[7] Harvey Brown. Physical relativity: space-time structure from a dynamical perspective. Oxford University Press, Oxford, 2005. 
[8] Harvey R. Brown and Christopher G. Timpson. Why special relativity should not be a template for a fundamental reformulation of quantum mechanics. In William Demopoulos and Itamar Pitowsky, editors, Physical Theory and its Interpretation: Essays in Honor of Jeffrey Bub. Springer, 2007.

[9] Jeffrey Bub. Von Neumann's projection postulate as a probability conditionalization rule in quantum mechanics. Journal of Philosophical Logic, 6:381-390, 1977.

[10] Jeffrey Bub. Quantum information and computation. In Handbook of Philosophy of Physics; arXiv e-print quant-ph/0512125. North-Holland, 2006.

[11] Jeffrey Bub. Quantum probabilities as degrees of belief. Studies in the History and Philosophy of Modern Physics, 38:232-254, 2007.

[12] William Demopoulos. Effects and propositions. Foundations of Physics, forthcoming, 2008.

[13] David Deutsch. Quantum theory of probability and decisions. Proceedings of the Royal Society of London, A455:3129-3137, 1999.

[14] Andrew Elby and Jeffrey Bub. Triorthogonal uniqueness theorem and its relevance to the interpretation of quantum mechanics. Physical Review A, 49:42134216, 1994.

[15] Christopher A. Fuchs. Quantum mechanics as quantum information (and only a little more). arXiv e-print quant-ph/0205039, 2002.

[16] Gian-Carlo Ghirardi. Collapse theories. In E.N. Zalta, editor, The Stanford Encyclopedia of Philosophy, 2002. http://plato.stanford.edu/entries/qm-collapse/.

[17] A.N. Gleason. Measures on the closed sub-spaces of Hilbert spaces. Journal of Mathematics and Mechanics, 6:885-893, 1957.

[18] Sheldon Goldstein. Bohmian mechanics. In E.N. Zalta, editor, The Stanford Encyclopedia of Philosophy, 2001. http://plato.stanford.edu/entries/qm-bohm.

[19] Hilary Greaves. Understanding Deutsch's probability in a deterministic multiverse. Studies in History and Philosophy of Modern Physics, 35:423-456, 2004.

[20] Hilary Greaves. On the Everettian epistemic problem. Studies in History and Philosophy of Modern Physics, 38:120-152, 2007.

[21] Hilary Greaves. Probability in the Everett interpretation. Philosophy Compass, 2:109-128, 2007.

[22] Meir Hemmo and Itamar Pitowsky. Quantum probability and many worlds. Studies in History and Philosophy of Modern Physics, 2007.

[23] Lane P. Hughston, Richard Jozsa, and William K. Wootters. A complete classification of quantum ensembles having a given density matrix. Physics Letters A, 183:14-18, 1993. 
[24] Michel Janssen. Drawing the line between kinematics and dynamics. Symposium Time and Relativity, Institute for Advanced Study, University of Minnesota, October 26, 2007; http://www.tc.umn.edu/ janss011/pdf\%20files/IASsymposiumF07.pdf, 2007.

[25] Simon Kochen and E.P. Specker. On the problem of hidden variables in quantum mechanics. Journal of Mathematics and Mechanics, 17:59-87, 1967.

[26] Itamar Pitowsky. Betting on the outcomes of measurements: a Bayesian theory of quantum probability. Studies in History and Philosophy of Modern Physics, 34:395-414, 2003.

[27] Itamar Pitowsky. Quantum mechanics as a theory of probability. In William Demopoulos and Itamar Pitowsky, editors, Festschrift in honor of Jeffrey Bub. Springer, Western Ontario Series in Philosophy of Science, New York, 2007. arXiv e-print quant-ph/0510095.

[28] Simon Saunders. Time, decoherence and quantum mechanics. Synthese, 102:235-266, 1995.

[29] Simon Saunders. Time, quantum mechanics, and probability. Synthese, 114:373404, 1998.

[30] Simon Saunders. Derivation of the born rule from operational assumptions. Proceedings of the Royal Society of London, A460:1-18, 2004.

[31] U. Uhlhorn. Representation of symmetry transformations in quantum mechanics. Arkiv Fysik, 23:307, 1963.

[32] John von Neumann. Unsolved problems in mathematics. In Miklós Rédei and Michael Stöltzner, editors, John von Neumann and the Foundations of Quantum Physics, pages 231-245. Kluwer Academic Publishers, Dordrecht, 2001.

[33] David Wallace. Everett and structure. Studies in History and Philosophy of Modern Physics, 34:86-105, 2003.

[34] David Wallace. Everettian rationality: defending Deutsch's approach to probability in the Everett interpretation. Studies in History and Philosophy of Modern Physics, 34:415-438, 2003.

[35] David Wallace. Quantum probability from subjective likelihood: improving on Deutsch's proof of the probability rule. arXiv e-print quant-ph/0312157, 2003.

[36] David Wallace. Epistemology quantized: circumstances in which we should come to believe in the Everett interpretation. British Journal for the Philosophy of Science, 2008.

[37] Eugene Wigner. Group Theory and its Applications to Quantum Mechanics of Atomic Spectra. Academic Press, New York, 1959. 
[38] Wojciech H. Zurek. Decoherence, einselection, and the quantum origins of the classical. Review of Modern Physics, 75:715, 2003.

[39] Wojciech H. Zurek. Probabilities from entanglement, Born's rule $p_{k}=\left|\psi_{k}\right|^{2}$ from envariance. Physical Review A, 71:052105-1-0525105-29, 2005. 